\title{
Circular photogalvanic effect induced by monopolar spin orientation in $p$-GaAs/AIGaAs multiple-quantum wells
}

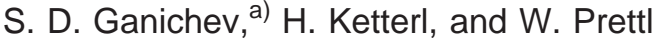 \\ Institut für Experimentelle und Angewandte Physik, Universität Regensburg, D93040 Regensburg, Germany \\ E. L. Ivchenko \\ A. F. Ioffe Physico-Technical Institute, Russian Academy of Science, 194021 St. Petersburg, Russia \\ L. E. Vorobjev \\ Petersburg State Technical University, 195251 St. Petersburg, Russia
}

(Received 31 May 2000; accepted for publication 27 September 2000)

\begin{abstract}
The circular photogalvanic effect (CPGE) has been observed in (100)-oriented $p$-GaAs/AlGaAs quantum wells at normal incidence of far-infrared radiation. It is shown that monopolar optical spin orientation of free carriers causes an electric current which reverses its direction upon changing from left to right circularly polarized radiation. CPGE at normal incidence and the occurrence of the linear photogalvanic effect indicate a reduced point symmetry of studied multilayered heterostructures. As proposed, CPGE can be utilized to investigate separately spin polarization of electrons and holes and the symmetry of quantum wells. (c) 2000 American Institute of Physics. [S0003-6951(00)05446-2]
\end{abstract}

Spin polarization and spin relaxation in semiconductors are the subject of intensive studies of spin-polarized electron transport aimed at the development of spinotronic devices like a spin transistor. ${ }^{1,2}$ So far, photoinduced spin polarization has been achieved by interband optical absorption of circularly polarized light with the photogeneration of spinpolarized electrons and holes. ${ }^{3-5}$

Here we report on the circular photogalvanic effect (CPGE) observed under intersubband transitions in multiplequantum well (MQW) structures. Phenomenologically, the effect is a transfer of angular momenta of circularly polarized photons into the directed movement of free carriers, electrons, or holes, and therefore depends on the symmetry of the medium. Microscopically, it arises due to optical spin orientation of holes in MQWs and asymmetric spindependent scattering of spin-polarized carriers by interface imperfections followed by an appearance of an electric current. The two states of light circular polarization $\sigma_{ \pm}$result in different spin orientations and, thus, in electric photocurrents of opposite directions. The CPGE has been observed on $p$-doped (001)-oriented MQWs at normal incidence of the circularly polarized far-infrared (FIR) laser pulses. In contrast to the case of interband optical excitation, under intersubband transitions only one kind of carrier is involved, leading to a monopolar spin orientation. The observed effect can be utilized to investigate spin polarization of extrinsic free carriers, and provides a simple method to analyze the reduced point symmetry of the medium under study, here MQWs.

The experiments have been carried out on $p$-GaAs/AlGaAs(001)-metalorganic chemical vapor deposition grown quantum well $(\mathrm{QW})$ structures with 400 undoped

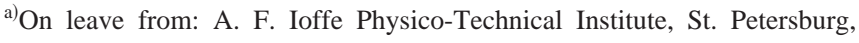
Russia; author to whom correspondence should be addressed; electronic mail: Sergey.Ganichev@physik.uni-regensburg.de wells of $20 \mathrm{~nm}$ width separated by $10 \mathrm{~nm}$ wide doped barriers. Different samples of $5 \times 5 \mathrm{~mm}^{2}$ size with surface hole densities $p_{s}$ in the QWs varying from $2 \times 10^{11}$ to 2 $\times 10^{12} \mathrm{~cm}^{-2}$ have been investigated. Ohmic contacts to the QWs were formed by depositing Ti and Au with subsequent tempering. Two pairs of point contacts along two perpendicular connecting lines have been prepared, parallel to [1 10$]$ and [110] (see inset in Fig. 1). Two additional pairs of ohmic contacts have been formed in the corners of the sample corresponding to the $\langle 100\rangle$ crystallographic directions.

A high power pulsed FIR molecular laser pumped by a transversly excited atmospheric pressure (TEA) $\mathrm{CO}_{2}$ laser has been used as a radiation source delivering $100 \mathrm{~ns}$ pulses with the intensity $I$ up to $1 \mathrm{MW} / \mathrm{cm}^{2}$. $\mathrm{NH}_{3}$ has been used as a FIR laser medium yielding strong single-line linearly polarized emissions at wavelengths $\lambda=76,90$, and $148 \mu \mathrm{m}$.

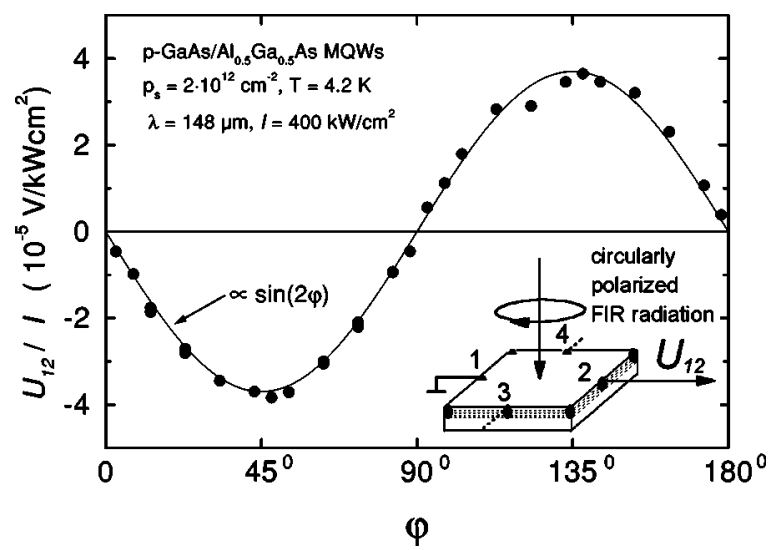

FIG. 1. Photogalvanic voltage signal $U_{12}$ picked up across the contact pair 1-2 and normalized by the intensity $I$ as a function of phase angle $\varphi$. The inset bottom-right shows the geometry of sample, contacts, and irradiation. The full line is fitted with one parameter after $U_{12} \propto P_{\text {circ }}$ [see first line in Eq. (3)]. The orientations of contact pairs $1-2$ and $3-4$ are along [1 $1 \overline{1} 0]$ and [110] crystallographic axes, respectively. 


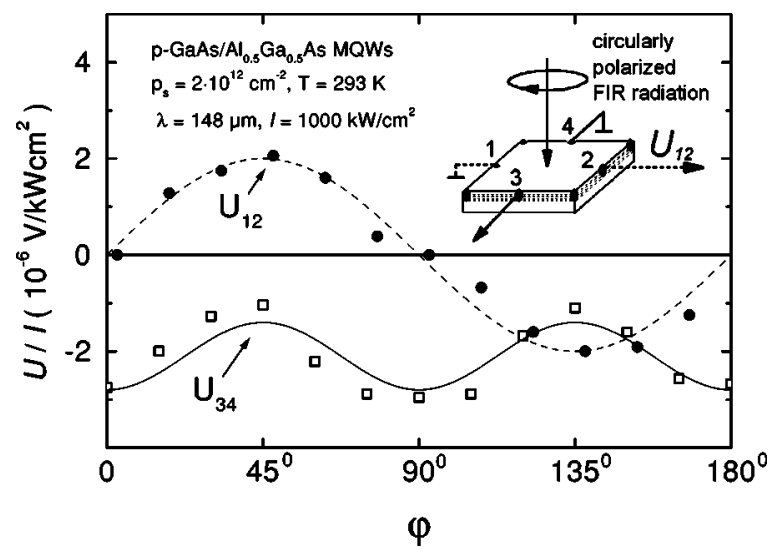

FIG. 2. Photogalvanic voltage signal $U\left[\right.$ (dots) $U_{12}$, (squares) $\left.U_{34}\right]$ normalized by the intensity $I$ for the two different contact pairs with orthogonal connecting lines (see inset) as a function of phase angle $\varphi$. The broken line and the full line are fitted after Eq. (3) as $U_{12} \propto \sin 2 \varphi$ and $U_{34} \propto\left(\chi_{+}\right.$ $\left.+\chi_{-} \cos ^{2} 2 \varphi\right)$, respectively.

The photon energies of the laser lines correspond to transitions between size-quantization subbands of GaAs QWs. ${ }^{8}$ The linearly polarized laser light could be modified to a circularly polarized radiation by applying crystalline quartz $\lambda / 4$ plates. As the experimental results were qualitatively the same for all three laser lines, measurements for one wavelength only $(148 \mu \mathrm{m})$ will be presented.

While irradiating the (001) GaAs QWs by normally incident circularly polarized radiation (see inset in Fig. 1) a fast photocurrent signal $U_{12}$ has been observed in unbiased samples across one contact pair (contacts 1 and 2 in the inset). The signal follows the temporal structure of the laser pulse intensity. The photocurrent changes sign if the circular polarization is switched from $\sigma_{+}$to $\sigma_{-}$. Measurements of $U_{12}$ as a function of the degree of circular polarization $P_{\text {circ }}$ $=\sin 2 \varphi$, where $\varphi$ is the angle between the optical axis of the $\lambda / 4$ plate and the plane of polarization of the laser radiation, reveal that the photogalvanic current $j$ is proportional to $P_{\text {circ }}$ (Fig. 1). In case of the linearly polarized radiation corresponding to $\varphi=0^{\circ}$ or $90^{\circ}$ the signal $U_{12}$ vanishes. The magnitude and the sign of the CPGE are practically unchanged with variation of the angle of incidence in the range of $-40^{\circ}-+40^{\circ}$. Heating the sample from 4.2 to $300 \mathrm{~K}$ leads to a change of the sign of CPGE but the $\sin 2 \varphi$ dependence is retained. In bulk GaAs substrate material no CPGE could be detected which gives evidence that the effect occurs in the MQWs.

Using the other pair of contacts (contacts 3 and 4 in the inset of Fig. 2) no photogalvanic signal dependent on the sign of $P_{\text {circ }}$ could be detected. This is demonstrated in Fig. 2 where the voltage $U_{34}$ is plotted as a function of the phase angle $\varphi$ together with $U_{12}$. The signal $U_{34}$ is periodic in $\varphi$ with the period being one half of that of $U_{12}$. The value and the sign of $U_{34}$ for $\sigma_{+}$are the same as that for $\sigma_{-}(\varphi=$ $\left.\pm 45^{\circ}\right)$. The measurements of the photogalvanic effect along $\langle 100\rangle$ crystallographic directions show a sum of the projections on this direction of the photocurrents induced along [11̄0] and [110].

The photogalvanic current under study can be described by the following phenomenological expression: ${ }^{9}$

$$
j_{\lambda}=\chi_{\lambda \mu \nu}\left(E_{\mu} E_{\nu}^{*}+E_{\nu} E_{\mu}^{*}\right) / 2+\gamma_{\lambda \mu} i\left(\mathbf{E} \times \mathbf{E}^{*}\right)_{\mu},
$$

where $\mathbf{E}$ is the complex amplitude of the electric field of the electromagnetic wave and

$$
i\left(\mathbf{E} \times \mathbf{E}^{*}\right)=P_{\text {circ }} E_{0}^{2} \frac{\mathbf{q}}{q},
$$

$E_{0}=|\mathbf{E}|$, and $\mathbf{q}$ is the light wave vector. In a bulk crystal, $\lambda=x, y, z$, while in a MQW structure grown along the $z$ direction, say $z \|[001]$, the index $\lambda$ runs only over the in-plane axes $x\|[100], y\|[010]$ because the barriers prevent carriers from moving along the $z$ axis and, definitely, $j_{z}=0$.

In bulk materials the photogalvanic effects under consideration arise in homogeneous samples under homogeneous excitation due to an asymmetry of the interaction of free carriers with photons, phonons, static defects, or other carriers in noncentrosymmetric media. ${ }^{9}$ The photocurrent given by the tensor $\boldsymbol{\chi}$ describes the so-called linear photogalvanic effect because it is usually observed under linearly polarized optical excitation. The CPGE described by the pseudotensor $\gamma$ can be observed only under circularly polarized optical excitation.

In an ideal (001)-grown MQW structure with the $D_{2 d}$ point symmetry or in a MQW structure with nonequivalent left and right interfaces yet retaining their local symmetry $C_{2 v}$, a generation of the circular photocurrent under normal incidence is forbidden because the pseudovector component $\left[\mathbf{E} \times \mathbf{E}^{*}\right]_{z}$ and the vector components $j_{x}, j_{y}$ transform according to nonequivalent irreducible representations of the group $D_{2 d}$ or $C_{2 v}$. Thus in order to explain the experimental data the symmetry of the structure should be reduced to the next possible point group which is $C_{s}$ and contains only two elements: the identity transformation and one mirror reflection, say in the plane $(1 \overline{1} 0)$. In this case we obtain under normal incidence

$$
\begin{aligned}
& j_{x^{\prime}}=E_{0}^{2}\left[\gamma P_{\text {circ }}+\chi_{1}\left(e_{x^{\prime}} e_{y^{\prime}}^{*}+e_{y^{\prime}} e_{x^{\prime}}^{*}\right)\right], \\
& j_{y^{\prime}}=E_{0}^{2}\left[\chi_{2}\left|e_{x^{\prime}}\right|^{2}+\chi_{3}\left|e_{y^{\prime}}\right|^{2}\right],
\end{aligned}
$$

where $\mathbf{e}$ is the polarization unit vector of the light and $\gamma, \chi_{1}$, $\chi_{2}$, and $\chi_{3}$ are linearly independent coefficients which can depend on the light frequency and temperature. Instead of $x$ and $y$ we use the axes $x^{\prime}\left\|[1 \overline{1} 0], y^{\prime}\right\|[110]$. It follows then that the circular photocurrent is induced along $x^{\prime}$, while the linear photocurrent induced by the light linearly polarized along $x^{\prime}$ or $y^{\prime}$ flows in the $y^{\prime}$ direction. For the light initially polarized along $x^{\prime}$ and transmitted through the $\lambda / 4$ plate we have

$$
\begin{aligned}
& j_{x^{\prime}}=\gamma E_{0}^{2} \sin 2 \varphi=\gamma E_{0}^{2} P_{\text {circ }}, \\
& j_{y^{\prime}}=E_{0}^{2}\left(\chi_{+}+\chi_{-} \cos ^{2} 2 \varphi\right)=E_{0}^{2}\left(\chi_{2}-\chi_{-} P_{\text {circ }}^{2}\right),
\end{aligned}
$$

where $\chi_{ \pm}=\left(\chi_{2} \pm \chi_{3}\right) / 2$. The CPGE described by $\gamma$ can be related to an electric current in a system of free carriers with nonequilibrium spin polarization. ${ }^{10}$ The possible microscopic mechanism is the spin-dependent scattering of optically oriented holes by asymmetric interface imperfections. The experimentally observed change of sign of the photogalvanic current upon heating the sample from $4.2 \mathrm{~K}$ to room temperature may be caused by the change of scattering mechanism from impurity scattering to phonon assisted scattering. Note that, for ideal zinc-blende-based MQW structures 


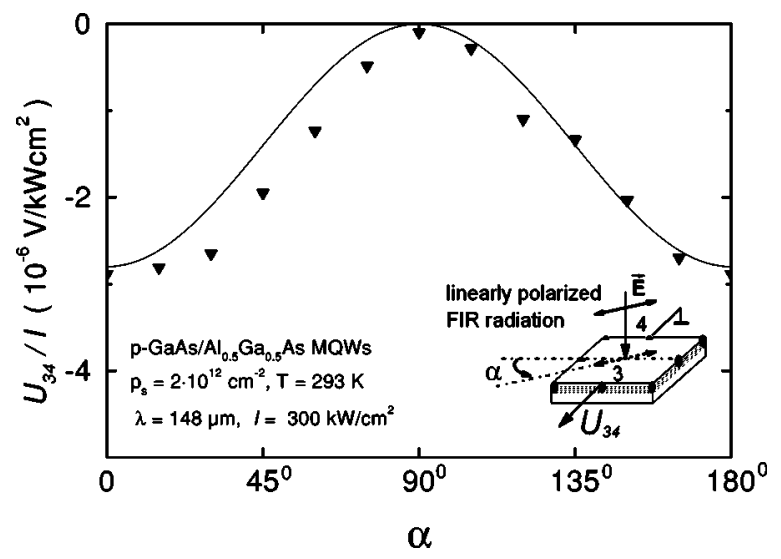

FIG. 3. Photogalvanic voltage signal $U_{34}$, in response to linear polarized radiation, picked up across the contact pair 3-4 and normalized by the intensity $I$ as a function of angle $\alpha$ between the electric field vector and the connecting line of contact pair 1-2 (see inset). Full line is fitted to $U_{34}$ $\propto\left[\chi_{+}-\chi_{-} \cos 2\left(\alpha+90^{\circ}\right)\right]$ where the same parameters $\chi_{+}$and $\chi_{-}$have been used as for fitting $U_{34}$ in Fig. 2.

grown along the principal axis [001], the CPGE is also possible but only under oblique incidence of irradiation.

Comparison of Eq. (3) and Figs. 1 and 2 demonstrates good agreement between the theory and the experimental data. In order to verify independently the validity of Eq. (2) for $j_{y^{\prime}}$ we excited the sample by linearly polarized light and measured the voltage $U_{34}$ as a function of the angle $\alpha$ between the plane of linear polarization and the axis $x^{\prime}$. Note, that in this setup $\alpha=0$ is equivalent to $\varphi=n 90^{\circ}$ ( $n$ is integer $0, \pm 1 \ldots)$ in the setup with the $\lambda / 4$ plate. The triangles in Fig. 3 present the measured dependence $U_{34}(\alpha)$, where the solid curve is calculated after $U_{34} \propto\left[\chi_{+}-\chi_{-} \cos 2\left(\alpha+90^{\circ}\right)\right]$ which follows from the second line of Eq. (2). To obtain the curve in Fig. 3 the same parameters were used as for fitting $U_{34}$ in Fig. 2.

The low symmetry $C_{s}$ required to have nonzero $\gamma$ and $\chi_{i}(i=1,2,3)$ gives evidence of the in-plane anisotropy, showing that in the investigated MQWs: (i) the [110] and [1 $1 \overline{1} 0]$ crystallographic directions are different, and (ii) one of the reflection planes, either (110) or (1 $1 \overline{1} 0)$, is removed as a symmetry element making the corresponding normal axis a polar one. The first conclusion is valid for both the $C_{2 v}$ and $C_{s}$ point groups; the difference between the [110] and [1 $\left.1 \overline{1} 0\right]$ axes has been studied by chemical etching ${ }^{11}$ of GaAs (001) surfaces and by an observation of the in-plane anisotropy of the quantum Hall effect ${ }^{12}$ and anisotropic exchange splitting of the excitonic levels (see Ref. 13 and references therein) in periodic heterostructures. Moreover, (001) faces of GaAs show trenches on the atomic level in one of the $\langle 110\rangle$ directions. ${ }^{14}$ Obviously these trenches proceed into MQWs forming interface islands, steps, etc., and reducing their symmetry. Conclusion (ii) is less obvious. It is worth noting that, unlike conventional optical and transport phenomena, the CPGE unambiguously chooses between the $C_{2 v}$ and $C_{s}$ symmetries. At present we can only guess why the available series of MQWs is characterized by the reduced symmetry. Photogalvanic studies of samples supplied from different sources are needed to come to a final conclusion.

To summarize, it is shown that the optical orientation of free carriers can be accompanied by their drift. Absorption of normally incident circularly polarized FIR radiation in gyrotropic media induces a photocurrent which reverses the direction as the circular polarization is changed from right- to left-hand orientation. These observations reveal the reduced in-plane symmetry of GaAs-based (001)-grown MQWs and can be used as a simple method to detect and characterize asymmetries in heterostructures. The method is effective even at room temperature. The theoretical considerations show that the CPGE can be observed also in an ideal MQW of the symmetry $D_{2 d}$ if the incident radiation is obliquely incident. The experiments presented here have been carried out in $p$-doped QWs but similar results are also expected for $n$-QWs.

Financial support by the DFG and the RFFI are gratefully acknowledged. The authors would like to thank H. v. Philipsborn for helpful discussions.

${ }^{1}$ S. Datta and D. Das, Appl. Phys. Lett. 56, 665 (1990).

${ }^{2}$ G. Prinz, Phys. Today 48, 58 (1995).

${ }^{3}$ Optical Orientation, edited by F. Meier and B. P. Zacharchenia (Elsevier, Amsterdam, 1984).

${ }^{4}$ J. M. Kikkawa and D. D. Awschalom, Nature (London) 397, 139 (1999).

${ }^{5}$ J. M. Kikkawa and D. D. Awschalom, Phys. Rev. Lett. 80, 4313 (1998).

${ }^{6}$ S. D. Ganichev, W. Prettl, and I. N. Yassievich, Phys. Solid State 39, 1703 (1997).

${ }^{7}$ S. D. Ganichev, Physica B 273-274, 737 (1999).

${ }^{8}$ L. E. Vorob'ev, D. V. Donetskii, and L. E. Golub, JETP Lett. 63, 977 (1996).

${ }^{9}$ E. L. Ivchenko and G. E. Pikus, Superlattices and Other Heterostructures, Springer Series in Solid State Sciences (Springer, Berlin, 1995).

${ }^{10}$ E. L. Ivchenko, Yu. B. Lyanda-Geller, and G. E. Pikus, Sov. Phys. JETP 71, 550 (1990).

${ }^{11}$ S. Adachi and K. Oe, J. Electrochem. Soc. 130, 2427 (1983).

${ }^{12}$ M. P. Lilly, K. B. Cooper, J. P. Eisenstein, L. N. Pfeiffer, and K. W. West, Phys. Rev. Lett. 82, 394 (1999).

${ }^{13}$ R. I. Dzhioev, H. M. Gibbs, E. L. Ivchenko, G. Khitrova, V. L. Korenev, M. N. Tkachuk, and B. P. Zakharchenya, Phys. Rev. B 56, 13405 (1997).

${ }^{14}$ R. E. Newnham, Structure-Property Relations (Springer, Heidelberg, 1975). 